\title{
ESTIMULAÇÃO ELÉTRICA NERVOSA TRANSCUTÂNEA NA DISMENORREIA PRIMÁRIA EM MULHERES JOVENS
}

\author{
TRANSCUTANEOUS ELECTRICAL NERVE STIMULATION IN PRIMARY DYSMENORRHEA IN YOUNG \\ WOMEN
}

\author{
Maria Carolina Torrilhas ${ }^{\mathrm{a}}$, Rafaela Dresch ${ }^{\mathrm{b}}$, Yngrid Haiany Monteiro de Oliveira Navarroc, \\ Márcia Rosângela Buzanello ${ }^{\mathrm{d}}$, Gladson Ricardo Flor Bertolini ${ }^{\mathrm{e}}$ \\ acatorrilhas@hotmail.com, brafaela_dresch9@hotmail.com, cyngridhmon@hotmail.com, dmrbuzanello@yahoo.com.br, \\ egladson_ricardo@yahoo.com.br \\ Universidade Estadual do Oeste do Paraná - Cascavel (PR), Brasil
}

Data de recebimento do artigo: 20/07/2017

Data de aceite do artigo: 07/08/2017

\section{RESUMO}

Introduçáo: A dismenorreia caracteriza-se pela dor abdominopélvica antes ou durante o período menstrual. Dor cíclica que pode ser acompanhada por cefaleia, vômito, tontura, sensação de inchaço, entre outros sintomas. Objetivo: Analisar o efeito analgésico da estimulação elétrica nervosa transcutânea (TENS) na dismenorreia primária em jovens nulíparas. Métodos: Participaram do estudo vinte voluntárias, com idade entre 18 e 26 anos. As participantes foram separadas aleatoriamente em dois grupos: grupo placebo (GP, $n=10)$ e grupo tratamento $(\mathrm{GT}, \mathrm{n}=10)$. A avaliaçấo da dor foi realizada por meio da escala visual analógica (EVA) e do questionário de dor de McGill. O procedimento diário teve duraçáo de 30 minutos, frequência de $100 \mathrm{~Hz}$ e duração de pulso $200 \rrbracket_{\mathrm{s}}$, intensidade forte, porém, confortável. Resultados: A redução média dos índices de dor foi maior para GT, porém ambos apresentaram redução significativa. Conclusáo: A TENS foi eficaz no tratamento da dor causada pela dismenorreia primária em jovens nulíparas, porém apenas ligeiramente diferente do placebo.

Palavras-chave: Dismenorreia; estimulação elétrica nervosa transcutânea; escala visual analógica; medição da dor.

\section{ABSTRACT}

Introduction: Dysmenorrhea is characterized by abdominopelvic pain before or during menstruation. Cyclic pain that may be accompanied by headache, vomiting, dizziness, bloating, and other symptoms. Objective: To evaluate the transcutaneous electrical nerve stimulation (TENS) analgesic effect for primary dysmenorrhea in young nulliparous women. Methods: The study included twenty volunteers, aged 18 to 26 years. The participants were randomized in two groups: placebo group (PG, $\mathrm{n}=10)$ and treatment group (TG, $\mathrm{n}=10)$. Pain assessment was performed by the visual analog scale (VAS) and the McGill pain questionnaire. The daily procedure was 30 minutes duration, frequency of $100 \mathrm{~Hz}$ and pulse duration $200 \mu$ s, strong intensity, but comfortable. Results: The mean reduction in pain levels were higher for TG, but for both groups they were significantly reduced. Conclusion: The TENS was effective in treating pain caused by primary dysmenorrhea in young nulliparous women, but only slightly different from placebo.

Keywords: Dysmenorrhea; transcutaneous electrical nerve stimulation; visual analog scale; pain measurement. 


\section{Introdução}

Dismenorreia, definida como cólica dolorosa que ocorre com a menstruação, é o problema ginecológico mais comum e uma das causas mais comuns de dor pélvica. Os sintomas geralmente iniciam na adolescência, podendo levar ao absenteísmo na escola e no trabalho, tanto quanto a limitaçôes sociais, acadêmicas e em atividades esportivas ${ }^{1}$. Provém do istmo uterino hipertônico, resultando em uma retenção temporária de resíduos do fluxo menstrual e causando grande pressão nessa área. A dor pode estar associada à isquemia em função da vigorosa contração dos músculos do útero ${ }^{2}$. Outros sintomas associados podem incluir lombalgia, cefaleia, diarreia, fadiga, náuseas e vômitos1. Apesar de ser comum, é subdiagnosticada e subtratada ${ }^{3}$.

Com base na patofisiologia, a dismenorreia pode ser subclassificada em primária ou secundária. A primária apresenta-se como dolorosa, com cólicas espasmódicas no baixo abdômen, logo antes e/ou durante a menstruação, na ausência de doença pélvica macroscópica discernível. O início geralmente ocorre na adolescência, logo após a menarca (6 a 24 meses). A dor tipicamente demora de 8 a 72 horas, geralmente no primeiro ou segundo dia da menstruação, e pode ser referida para a regiáo lombar e coxas. A dismenorreia secundária origina-se de uma condiçáo patológica identificável, como endometrioses, adenomioses, miomas e doenças inflamatórias pélvicas ${ }^{3}$.

A prevalência varia entre 16 e $91 \%$ em mulheres em idade reprodutiva, sendo que entre 2 e $29 \%$ apresentam dor considerada grave. $\mathrm{O}$ avanço da idade e do número de filhos e o uso de contraceptivos orais são inversamente associados com dismenorreia, enquanto altos níveis de estresse aumentam o risco de dismenorreia, bem como histórico familiar ${ }^{4}$. A explicação mais aceita para a patogênese de dismenorreia primária é a superprodução de prostaglandinas uterinas. Acreditase causar hipercontratilidade miometrial, resultando em isquemia, hipóxia da musculatura uterina e, finalmente, dor ${ }^{3}$.

Alguns recursos fisioterapêuticos são utilizados, visando produzir alívio dos sintomas da dismenorreia, sendo que alguns parecem não ter efeitos maiores do que o placebo, como a acupuntura e acupressão, porém, outros como o calor local, a estimulação elétrica nervosa transcutânea (TENS) 5 e o protocolo de exercícios (fortalecimento, alongamento e relaxamento muscular) $^{6}$ mostram-se como eficazes instrumentos para alívio dos sintomas.

Para o TENS ainda existem controvérsias com relação aos seus efeitos, contudo há indícios de que este seja efetivo, tanto em alta quanto em baixa frequência, no alívio dos sintomas da dismenorreia ${ }^{7}$, mas para seu uso outros parâmetros também apresentam-se importantes, como o local de estímulo, a intensidade ${ }^{8}$ e até mesmo a duração de fase ${ }^{9}$. Sendo assim, a proposta deste estudo foi analisar o efeito de um específico protocolo da TENS como forma de tratamento da dor gerada pela dismenorreia primária em jovens nulíparas.

\section{Materiais e métodos}

Este estudo caracteriza-se como quantitativo e experimental. A amostra, inicialmente composta por trinta universitárias de uma instituiçáo pública, foi eleita por conveniência e de forma não probabilística. Vinte voluntárias atenderam aos critérios de inclusão: idade entre 18 e 26 anos, com dismenorreia primária e que não fizessem nenhum tipo de eletroestimulação como tratamento no período em que participariam do estudo. Adotou-se como critérios de exclusão: gestação, problemas cardíacos e outras contraindicaçóes ao uso da eletroestimulação. A amostra final de 20 indivíduos foi aleatoriamente distribuída em dois grupos $(\mathrm{n}=10)$ : grupo placebo (GP) e grupo tratamento (GT) (Figura 1). A pesquisa foi aprovada pelo Comitê de Ética em Pesquisa da Unioeste (CAAE: 46189415.5.0000.0107).

Figura 1: Fluxograma representativo do estudo baseado no CONSORT

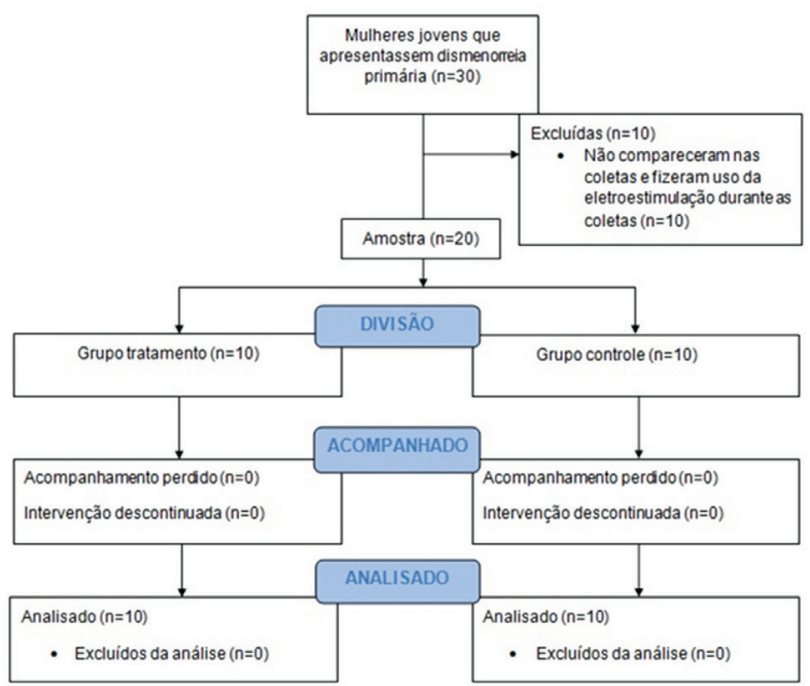

Fonte: Baseado no modelo apresentado em no Fluxograma CONSORT 2010. Elaborado pelos autores, 2017

A coleta foi realizada entre o segundo e quarto dia do ciclo menstrual. Previamente foram aplicados o 
questionário McGill (índice de dor), adaptado para o português ${ }^{10}$, e a escala visual analógica (EVA). Ainda foi questionado em anamnese dados sobre menarca e sintomas associados.

Inicialmente, aplicou-se o questionário McGill no início da primeira intervenção e no último dia após o procedimento, dessa forma, registrando-se a localização, a intensidade e o comportamento da dor nas dimensões sensitiva, afetiva e avaliativa. Em seguida, a EVA foi aplicada antes e após cada aplicação do TENS. Para isso, utilizou-se um dispositivo de madeira, graduado, em que para a voluntária aparecia apenas um escalímetro e as marcaçóes de 0 e 10 , sendo explicado que zero significava "sem dor"; o meio da escala "dor moderada"; e dez "a pior dor imaginável", e que a participante deveria indicar, por meio de um escalímetro, qual era a intensidade momentânea da dor. Para o avaliador, o escalímetro apontava em escala centimetrada a distância de $10 \mathrm{~cm}$, mostrando assim a graduação da dor.

O GT foi submetido ao protocolo de eletroestimulação diariamente (entre o segundo e quarto dia do ciclo menstrual), o qual consistiu em posicionar as voluntárias em decúbito ventral e fazer assepsia do local em que os eletrodos foram dispostos, com álcool 70\%. Em seguida, foi realizada a colocaçáo de quatro eletrodos (de borracha de silicone, com $8 \mathrm{~cm}^{2}$ ), que foram distribuídos paralelamente à coluna vertebral (distantes cerca de $3 \mathrm{~cm}$ do processo espinhoso) e entre as vértebras L3 e S3, acoplados à pele com uma camada de gel e fixados com fita crepe. Outros parâmetros de uso foram: duração de trinta minutos, frequência de $100 \mathrm{~Hz}$ e duração de pulso $200 \rrbracket \mathrm{s}$, com intensidade forte, porém não dolorosa, sendo uma terapia diária durante três dias.

O GT foi submetido a procedimento semelhante, porém o equipamento permaneceu desligado durante todo o tempo, sendo sugestionado à paciente que esta receberia apenas uma corrente com intensidade muito baixa.

Os dados foram avaliados, quanto a sua normalidade, pelo teste de Shapiro-Wilk, apresentados em média e desvio-padráo, comparados dentro dos grupos pelo teste t pareado e entre os grupos pelo teste t não-pareado, em todos os casos o nível de significância aceito foi de $5 \%$.

\section{Resultados}

A média de idade da menarca foi 12,8 anos $\pm 1,86$ anos. As principais queixas associadas ao período menstrual foram de dor lombar, cefaleia, náusea, tontura e diarreia, podendo estas estarem inter-relacionadas (Tabela 1).

Tabela 1: Principais queixas associadas ao período menstrual.

\begin{tabular}{lc} 
Principais queixas & Frequência (\%) \\
\hline Dor lombar & $10 \%$ \\
\hline Cefaleia & $40 \%$ \\
Diarreia & $5 \%$ \\
Tontura & $5 \%$ \\
Outros & $40 \%$ \\
\hline
\end{tabular}

Fonte: Elaborado pelos autores, 2017.

Para análise dos resultados obtidos com a EVA, calculou-se a média dos dias em que a dor prevaleceu e obteve-se sua intensidade. Ao analisar os dados, percebe-se uma redução significativa em relação à intensidade de dor referida pelas participantes do grupo tratamento (redução de 40,47\%; $\mathrm{p}=0,0024$ ) e do grupo placebo (redução de $30,5 \% ; \mathrm{p}=0,0067$ ), porém também existiam diferenças entre os grupos inicialmente (Tabela 2).

Tabela 2: Representação numérica dos valores obtidos para escala visual analógica de dor (EVA), para os grupos placebo e tratado com TENS, com o valor de significância obtido $(p)$, no início (i) e fim (f) das terapias.

\begin{tabular}{lccc} 
& Placebo & Tratamento & p \\
EVA i & $1,8 \pm 1,5$ & $4,9 \pm 2,2$ & $0,0019^{*}$ \\
EVA f & $1,2 \pm 1,4$ & $2,6 \pm 1,8$ & 0,0766 \\
p & $0,0067^{*}$ & $0,0024^{*}$ & \\
\hline
\end{tabular}

*Diferença significativa $(\mathrm{p}<0,05)$.

Fonte: Elaborado pelos autores, 2017.

Para o questionário de McGill, novamente houve redução significativa da dor depois do tratamento tanto para o grupo tratamento (redução de 44\%; p <0,0001) quanto para o grupo placebo (redução de 25,5\%; $\mathrm{p}=0,0161$ ), sem diferenças entre os grupos (Tabela 3 ).

Tabela 3: Representação numérica dos valores obtidos para Questionário de McGill, para os grupos placebo e tratado com TENS, com o valor de significância obtido (p), no início (i) e fim (f) das terapias.

\begin{tabular}{lccc} 
& Placebo & Tratamento & p \\
MCGILL i & $35,2 \pm 9,3$ & $42,3 \pm 10,2$ & 0,1224 \\
MCGILL f & $26,2 \pm 8,3$ & $23,7 \pm 8,1$ & 0,503 \\
$\mathrm{p}$ & $0,0161^{*}$ & $<0,0001^{*}$ & \\
\hline
\end{tabular}

*Diferença significativa $(\mathrm{p}<0,05)$.

Fonte: Elaborado pelos autores, 2017. 


\section{Discussão}

A dismenorreia primária é uma das queixas ginecológicas mais comuns entre mulheres jovens ${ }^{3}$, responsável por consideráveis perdas econômicas devido aos custos de medicaçóes e de cuidados médicos e à diminuiçáo da produtividade ${ }^{4}$. A menarca ao redor de 12 anos de idade apresenta correlaçáo com a gravidade da dismenorreia ${ }^{11}$. Neste estudo, a média da menarca das pacientes foi 12,8 anos, mas todas apresentaram essa disfunção de intensidade leve a moderada. Ao analisar o estudo, constatou-se que $80 \%$ das jovens participantes fazem uso de medicamento para analgesia (dados não apresentados). Em estudo realizado por Nunes et al. ${ }^{12}$, essa taxa foi de $81,3 \%$.

Visto que mulheres com dismenorreia primária tendem a apresentar menor nível de atividade física, diminuição da produtividade no trabalho e redução na qualidade de vida, medidas que avaliem seus sintomas são fundamentais, tendo-se à disposiçáo diversos instrumentos como a escala visual analógica (EVA) de dor, que é de fácil utilização e mede a intensidade da dor. Contudo uma grande limitação de medidas unidimensionais é o fato de elas não serem projetadas para capturar toda a sintomatologia da dismenorreia primária, tais como componentes afetivos, qualidade e aspectos temporais não são considerados. Outro importante instrumento é o questionário de McGill, que oferece uma avaliação mais completa, medindo não apenas o componente sensitivo da dor, mas também o afetivo e o avaliativo; contudo, tal ferramenta também apresenta problemas, pois náo captura a sintomatologia completa em mulheres com dismenorreia primária, e alguns de seus itens podem não ser relevantes, além de demandar tempo e atenção ${ }^{13}$.

O uso de equipamentos de TENS tem sido apontado como aliado no tratamento da dor produzida em casos de dismenorreia ${ }^{5,7}$. Deve-se levar em consideração que essa forma de terapia pode agir nas vias de controle da dor, tanto ascendentes quanto descendentes, dependentes de combinaçóes de parâmetros ${ }^{8}$. Assim, não teria efeitos diretos sobre a elevação nos níveis de prostaglandinas, que causam hipercontratilidade miometrial, resultando em isquemia e hipóxia da musculatura uterina e, por fim, dor ${ }^{3}$, nem alterações na pressão intrauterina ${ }^{14}$.

Para avaliar os efeitos da TENS de alta frequência na redução do quadro álgico de mulheres com dismenorreia primária, comparados com os do grupo placebo, este estudo utilizou vinte voluntárias divididas de forma aleatória. Os parâmetros utilizados na terapia do grupo tratamento foram frequência de $100 \mathrm{~Hz}$, duraçáo de pulso de $200 \bigotimes_{s}$ e intensidade adaptada à subjetividade da participante, pois solicitou-se que esta a referisse como intensa, porém não dolorosa. A duração da eletroestimulação foi de 30 minutos, e houve aumento na intensidade do estímulo elétrico quando as pacientes referiam ter ocorrido a acomodação. Para a aplicação, as voluntárias foram posicionadas em decúbito ventral, utilizando dois pares de eletrodos posicionados na região lombossacral.

Diversos estudos têm avaliado o equipamento de TENS frente à dismenorreia primária, contudo os parâmetros de estimulação não são uniformes. No estudo de Oliveira et al. ${ }^{15}$, que compararam os efeitos da TENS de alta e baixa frequência no alívio da dor em 15 mulheres com dismenorreia primária, as sessões de eletroestimulação foram de 30 minutos, com o aumento da intensidade do estímulo elétrico guiado pelas participantes. A duração de pulso foi de $100 \bigotimes_{s}$ para ambos os grupos, já a frequência foi de 100 ou $4 \mathrm{~Hz}$, os eletrodos $\left(15 \mathrm{~cm}^{2}\right)$ foram posicionados entre L3-4. Mesmo com a duração de pulso relativamente baixa, relatam diminuição da dor em ambos os grupos, porém diferente do resultado apresentado neste estudo; para o grupo placebo não houve tal reduçáo. Já Lundeberg et al. ${ }^{16}$, também compararam a estimulação de alta frequência $(100 \mathrm{~Hz})$ com baixa frequência $(2 \mathrm{~Hz})$, utilizando duraçáo de $200 \mu$ s e eletrodos com $36 \mathrm{~cm}^{2}$ em posiçóes variadas, de acordo com a analgesia apresentada. Os autores relatam que 14 das 21 pacientes apresentaram redução da dor acima de $50 \%$ quando estimuladas com alta frequência, já para baixa frequência foram 7 e para o placebo 5 referiram tal efeito.

Milsom et al. ${ }^{14}$ compararam a TENS de alta intensidade com naproxeno. A duração de fase utilizada foi de $200 \rrbracket \mathrm{s}$, frequência variável entre 70 a $100 \mathrm{~Hz}$, estimulando tanto a regiáo lombar quanto a regiáo abdominal baixa, porém a intensidade utilizada era alta (entre $40-50 \mathrm{~mA}$ ). Os autores relatam que apenas a droga produziu alteraçóes sobre a pressão uterina, mas o TENS foi capaz de reduzir a intensidade da dor. Também utilizando alta intensidade $(50 \mathrm{~mA})$, Wang et al. ${ }^{17}$, com TENS variando entre $100-120 \mathrm{~Hz}$ e $200 \bigotimes_{\mathrm{s}}$ de duração de pulso, observaram em 22 mulheres que a eletroestimulação foi benéfica, porém, para a intensidade da dor, o grupo placebo também apresentou diminuição dos valores.

Existem ainda estudos que apresentam formas não convencionais da TENS, como Lauretti et al. ${ }^{18}$, que apresentaram um dispositivo portátil, aplicado na região suprapúbica em 40 voluntárias, por 30 minutos, com 8 horas de intervalo, durante 7 dias, com frequência de $85 \mathrm{~Hz}$ e quatro possibilidades de nível de estimulação $(1-$ zero; $2-10 \mathrm{~mA} ; 3-20 \mathrm{~mA} ; 4-30 \mathrm{~mA})$, 
e observaram alívio da dor, sem efeitos colaterais. Semelhante ao observado por Lee et al. ${ }^{19}$ com um eletroestimulador combinado com calor, para os quais os parâmetros eram frequência variável entre $100-110 \mathrm{~Hz}$ por 10 minutos, seguido por termoterapia de 37 ou $40^{\circ}$ (dependendo das preferências individuais) por $20 \mathrm{mi}$ nutos. Ao analisar os resultados obtidos, notou-se que a utilização da terapia TENS nos parâmetros propostos demonstrou resultados positivos em relaçáo à diminuição da dor. Entretanto, apesar de a estatística descritiva apresentar melhores resultados para o grupo eletroestimulado, a estatística inferencial apenas demonstrou que a diferença inicial não ocorria ao final do experimento para a EVA. Tais resultados podem denotar possível interferência do efeito placebo, proporcionado pela intervenção projetada similar à intervenção eletroterapêutica.

De acordo com Rocha e Coelho ${ }^{20}$, define-se placebo como qualquer tratamento desprovido de ação terapêutica específica sobre a doença ou os sintomas do paciente, mas que de alguma forma provoca efeito sobre este, desde que o paciente esteja convencido da sua eficácia.

Dessa forma, existem vários estudos que comprovam a eficácia da eletroestimulaçáo tanto de alta como de baixa frequência sobre a dor gerada pelos transtornos da dismenorreia, sendo que mesmo a mudança de parâmetros como frequência, duração de fase, intensidade e local de aplicação parece não influenciar muito nos resultados. Vale ressaltar que este estudo tem como grande limitação a não estratificação inicial das voluntárias com relação à intensidade da dor, pois os grupos inicialmente eram diferentes, além do curto prazo de tempo avaliado (apenas 1 ciclo). Assim, sugere-se que tais cuidados possam ser tomados em futuros estudos que avaliem recursos físicos aplicados à dismenorreia primária.

\section{Conclusão}

Conclui-se com este estudo que a estimulação elétrica nervosa transcutânea apresentou redução do quadro álgico em mulheres com dismenorreia primária, contudo, foi apenas ligeiramente diferente do placebo.

\section{Referências}

1. Osayande AS, Mehulic S. Diagnosis and initial management of dysmenorrhea. Am Fam Physician. 2014;89(5):341-6.

2. Peruzzo BCT, Ramalho LS, Figueiredo MR, Alfieri FM. Benefícios sobre a intensidade da dor, qualidade de vida e incapacidade de mulheres com dismenorreia submetidas a exercícios gerais versus método de Pilates: estudo-piloto. ABCS Health Sci. 2015;40(1):6-10.

3. Iacovides S, Avidon I, Baker FC. What we know about primary dysmenorrhea today: a critical review. Hum Reprod Update. 2015;21(6):762-78.

4. Ju H, Jones M, Mishra G. The prevalence and risk factors of dysmenorrhea. Epidemiol Rev. 2014;36(1):104-13.

5. Kannan P, Claydon LS. Some physiotherapy treatments may relieve menstrual pain in women with primary dysmenorrhea: a systematic review. J Physiother. 2014;60(1):13-21.

6. Ortiz MI, Cortés-Márquez SK, Romero-Quezada LC, Murguía-Cánovas G, Jaramillo-Díaz AP. Effect of a physiotherapy program in women with primary dysmenorrhea. Eur J Obstet Gynecol Reprod Biol. 2015;194:24-9.

7. Gerzson LR, Padilha JF, Braz MM, Gasparetto A. Physiotherapy in primary dysmenorrhea: literature review. Rev Dor. 2014;15(4):290-5.

8. Claydon LS, Chesterton LS, Barlas P, Sim J. Dose-specific effects of transcutaneous electrical nerve stimulation (TENS) on experimental pain: a systematic review. Clin J Pain. 2011;27(7):635-47.

9. Doucet BM, Lam A, Griffin L. Neuromuscular electrical stimulation for skeletal muscle function. Yale J Biol Med. 2012;85(2):201-15.

10. Pimenta CAM, Teixeira MJ. Questionário de dor McGill: proposta de adaptaçáo para a língua portuguesa. Rev Bras Anestesiol. 1997;47(2):177-86.

11. Balbi C, Musone R, Menditto A, Di Prisco L, Cassese E, D'Ajello $\mathrm{M}$, et al. Influence of menstrual factors and dietary habits on menstrual pain in adolescence age. Eur J Obstet Gynecol Reprod Biol. 2000;91(2):143-8.

12. Nunes JMO, Rodrigues JA, Moura MSF, Batista SRC, Coutinho SKSF, Hazime FA, et al. Prevalence of dysmenorrhea in university students and its relation to school absenteeism, physical exercise and use of medicines. Rev Bras Promoç Saúde. 2013;26(3):374-9.

13. Chen CX, Kwekkeboom KL, Ward SE. Self-report pain and symptom measures for primary dysmenorrhoea: a critical review. Eur J Pain. 2015;19(3):377-91.

14. Milsom I, Hedner N, Mannheimer C. A comparative study of the effect of high-intensity transcutaneous nerve stimulation and oral naproxen on intrauterine pressure and menstrual pain in patients with primary dysmenorrhea. Am J Obstet Gynecol. 1994;170(1 Pt 1):123-9.

15. Oliveira RGCQ, Silva JC, Almeida AF, Araújo RC, Pitangui ACR. TENS de alta e baixa frequência para dismenorreia primária: estudo preliminar. Conscientiae Saúde. 2012;11(1):149-58.

16. Lundeberg T, Bondesson L, Lundström V. Relief of primary dysmenorrhea by transcutaneous electrical nerve stimulation. Acta Obstet Gynecol Scand. 1985;64(6):491-7.

17. Wang SF, Lee JP, Hwa HL. Effect of transcutaneous 
electrical nerve stimulation on primary dysmenorrhea. Neuromodulation. 2009;12(4):302-9.

18. Lauretti GR, Oliveira R, Parada F, Mattos AL. The new portable transcutaneous electrical nerve stimulation device was efficacious in the control of primary dysmenorrhea cramp pain. Neuromodulation. 2015;18(6):522-7.

19. Lee B, Hong SH, Kim K, Kang WC, No JH, Lee JR, et al. Efficacy of the device combining high-frequency transcutaneous electrical nerve stimulation and thermotherapy for relieving primary dysmenorrhea: a randomized, single-blind, placebo-controlled trial. Eur J Obstet Gynecol Reprod Biol. 2015;194:58-63.

20. Rocha MG, Coelho R. Placebo - compreender a cura pelo nada. Rev Port Psicossomática. 2003;5(2):141-54.

Como citar este artigo:

Torrilhas MC, Dresch R, Navarro YHMO, Buzanello MR, Bertolini GRF. Estimulação elétrica nervosa transcutânea na dismenorreia primária em mulheres jovens. Rev. Aten. Saúde. 2017;15(54):61-66. 